\title{
RNA-Sequencing-Based IncRNA Biomarker Profiling on Triple Negative Breast Cancer
}

\author{
Lin Gao ${ }^{1,2}$, Jinquan Xia ${ }^{1}$, Malin Hong ${ }^{1}$, Jingyi Huang ${ }^{1}$, Pan Zhao ${ }^{1,5}$, Li Fu ${ }^{6}$, Jigang Wang ${ }^{1.5}$, \\ Yong Dai ${ }^{1}$, Wenbin Zhou ${ }^{3}$, Qinhe Yang ${ }^{4, *}$ and Chang Zou ${ }^{1,5,{ }^{*}}$
}

${ }^{1}$ Department of Clinical Medical Research Center, The Second Clinical Medical College, Jinan University (Shenzhen People's Hospital); The First Affiliated Hospital of Southern University of Science and Technology, Shenzhen 518000, Guangdong, P.R. China

${ }^{2}$ Integrated Chinese and Western Medicine Postdoctoral Research Station, Jinan University, Guangzhou, Guangdong, P.R. China

${ }^{3}$ Department of Thyroid and Breast Surgery, The Second Clinical Medical College, Jinan University (Shenzhen People's Hospital); The First Affiliated Hospital of Southern University of Science and Technology, Shenzhen 518000, Guangdong, P.R. China

${ }^{4}$ Department of Integrated Chinese and Western Medicine, Jinan University, Guangzhou, Guangdong, P.R. China

${ }^{5}$ Shenzhen Public Service Platform on Tumor Precision Medicine and Molecular Diagnosis, the Second Clinical Medical College, Jinan University (Shenzhen People's Hospital), Shenzhen, Guangdong, PR China

${ }^{6}$ Guangdong Provincial Key Laboratory of Regional Immunity and Diseases, Department of Pharmacology and International Cancer Center, Shenzhen University Health Science Center, Shenzhen 518060, Guangdong, China

\begin{abstract}
Basal-like triple-negative breast cancer (TNBC) is highly heterogeneous and lack of effective molecular targets for therapy. In this study, we developed the IncRNA signatures of TNBC as molecular biomarkers. RNAsequencing in 12 paired breast cancer and adjacent tissues identified up-regulated and down-regulated IncRNAs of Basal subtype in contrast to Luminal A, Luminal B and HER2 subtypes. Additionally, Kaplan-Meier analysis revealed that high expression of IncRNA (ZEB1-AS1 and TMEM254-AS1) had a poor relapse-free survival rate (RFS), while high expression of IncRNA (LINC01087, LINC01122 and LINC00856) had a positive correlation with RFS. Furthermore, qRTPCR analysis showed that the mRNA expressions of the ZEB1-AS1 and TMEM254-AS1 IncRNA were up-regulated in TNBC tissues, while the mRNA expression of IncRNA, including LINC01087, LINC01122 and LINC00856 were downregulated in TNBC tissues. Taken together, our results elucidated that 5 novel IncRNAs, including ZEB1-AS1, TMEM254-AS1, LINC01087, LINC01122 and LINC00856 contributed to the progression of invasive TNBC. These IncRNAs could be molecular biomarkers for the development of TNBC treatment.
\end{abstract}

Keywords: TNBC, long noncoding RNA (InCRNA), RNA-sequencing, ZEB1-AS1, TMEM254-AS1, LINC01087, LINC01122, LINC00856.

\section{INTRODUCTION}

Targeted therapy of breast cancer (BCa) has achieved great advance in the past decades [1], however, $\mathrm{BCa}$ with the potential invasiveness and metastasis remains a major challenge for treatment. Triple negative breast cancer (TNBC) is defined that it does not express the estrogen receptor (ER), the progesterone receptor (PR) and human epidermal growth factor receptor 2 (Her2) proteins. TNBC is generally more aggressive and easily metastasized due to tumor heterogeneous than other types of breast

*Address correspondence to these authors at the Department of Integrated Chinese and Western Medicine, Jinan University, Guangzhou, Guangdong, P.R. China; E-mail: tyangqh@jnu.edu.cn

Shenzhen Public Service Platform on Tumor Precision Medicine and Molecular Diagnosis, the Second Clinical Medical College, Jinan University (Shenzhen People's Hospital), Shenzhen, Guangdong, PR China;

E-mail: zou.chang@szhospital.com cancer [2]. The incidence and mortality of TNBC are increasing recently. Though standard-of-care chemotherapy is applied, a part of the patients still has not obviously responded to treatment. It has been a long-lasting challenge that cancer heterogeneity of TNBC and the lack of better molecular targets [3]. Therefore, it is necessary to exploit novel and effective biomarkers for TNBC patients on personalized treatment.

Long noncoding RNAs (IncRNA) are a kind of noncoding RNA, and the lengths of RNA are more than 200 nucleotides. LncRNA may involve in cancer progression. Most of IncRNA cannot translate into proteins due to lacking significant open reading frames. However, some research also has been reported that IncRNA is able to encode small peptides. Huang et al. suggested that the conserved 53-aa small peptide was

(C) 2021 Neoplasia Research 
encoded by IncRNA HOXB-AS3, that was a suppressor of colon cancer and could regulate aerobic glycolysis by modulating PKM splicing [4]. In breast cancer, IncRNA is found to promote epithelial-mesenchymal transition and metastasis through competing with miRNAs at binding sites of target genes [5]. In addition, IncRNA DSCAM-AS1 was a potentially ER positive modulated IncRNA in acquired endocrine therapyresistant patients of $\mathrm{BCa}$ [6], that provided novel insight into anti-oestrogen therapy. The high expression of IncRNA HOTAIR was found to accelerate the aggressiveness of $\mathrm{BCa}$ tumor through regulating the expression of targets [7]. However, it still lacks an effective approach to develop key molecular targets for TNBC. Thus, it would have substantial value to screen effective targets for TNBC patients.

In the present study, we aimed to exploit and identify availably prognostic IncRNA from RNA sequencing. We developed the biomarkers of IncRNA that were driven by TNBC drawing from patient tissues RNA-seq cohort. Our findings could open an avenue in both comprehending the molecular basis of TNBC progression and developing novel treatment strategies overcoming this disease.

\section{MATERIALS AND METHODS}

\section{Ethics Statement}

All the use of surgical samples of BCa tissues was approved by the Institutional Review Board of the Second Clinical Medicine College of Jinan University (LL-KY-2019435).

\section{Breast Cancer Subtypes and Survival Analysis}

The BCa tissues and tumor adjacent tissues were obtained from patients diagnosed between 2019 and 2021 at Shenzhen People's Hospital (including 3 Luminal A, 3 Luminal B, 3 Her2 and 3 TNBC subtypes). The differential analysis of genes was obtained by edger analysis between the 3 basal (TNBC) samples of patients and 9 other subtype sample of $\mathrm{BCa}$ data [8]. The significant difference was $P$-value $<0.05$ and the threshold $\log _{2}[$ fold change] $\geq 2$. Kaplan-Meier survival curve for the outcome of BCa patients were performed using Kaplan-Meier database (www.kmplot.com).

\section{Quantitative Reverse Transcription-PCR (qRT-PCR)}

Total RNA was isolated from $\mathrm{BCa}$ tissues using RNeasy mini kit (QIAGEN) according to the manufacturer's instructions. Reverse transcription was performed using TransScript One-Step RT-PCR SuperMix (Transgen Biotech), and $1 \mu \mathrm{g}$ of RNA was used to produce cDNA according to the manufacturer's instructions. The BIO-RAD CFX96 Real-Time System (BIO-RAD) was utilized for qRT-PCR reactions. QRTPCR primers were designed by NCBI (https://www.ncbi.nlm.nih.gov/) and Integrated DNA Technologies (https://sg.idtdna.com/pages). All sequences of primers were found in Supplementary Table S1. QRT-PCRs were performed using SYBR Green PCR Master Mix (Thermo Fisher Scientific). The mRNA relative expression was analysed by the comparative $\mathrm{Ct}$ values. Three replicates were performed in each assay.

\section{RNA-Sequencing}

Total RNA was extracted from human $\mathrm{BCa}$ tissues and tumor-adjacent tissues. The concentration of RNA was qualitied by Qubit system, and the purity of RNA was detected by Nanodrop system (OD260/280), and the integrity of RNA was performed by Agilent 2100 system. The library was established by NEB library. RNA sequencing was performed at Beijing Novogene, using the Illumina Novaseq 6000 platform.

\section{Statistical Analysis}

All statistical analyses were performed according to SPSS 21.0 software. Relapse-free survival curves were generated by the Kaplan-Meier plotter and analysed using the long-rank test. QRT-PCR results were showed as the mean \pm standard deviation (SD). All data were analysed for a normal distribution and homogeneity of variance. Means were compared using independent-samples T-tests or One-way analysis of variance (ANOVA). A ${ }^{*} P<0.05,{ }^{* *} P<0.01,{ }^{* *} P<0.001$.

\section{RESULTS}

\section{Identification of Differential Expression of Genes in Different Subtypes of BCa}

We evaluated the differential expression of genes by RNA-seq. BCa samples were classified as 4 subtypes based on clinical histopathologic diagnosis. These subtypes contained the following: (1) Luminal A subtype featured by positive estrogen receptor and progestogen receptor and negative Her2 receptor; (2) Luminal B subtype featured by positive estrogen receptor and progestogen receptor and Her2 receptor; (3) Her2 subtype featured by positive human epithelial factor receptor; (2) TNBC (Basal-like) featured by negative estrogen receptor and progestogen receptor 
A

LuminalA Ca-VS-LuminalA P

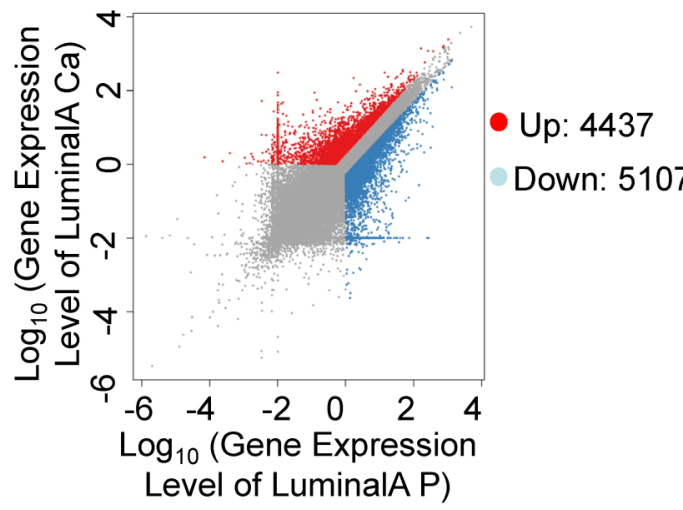

C

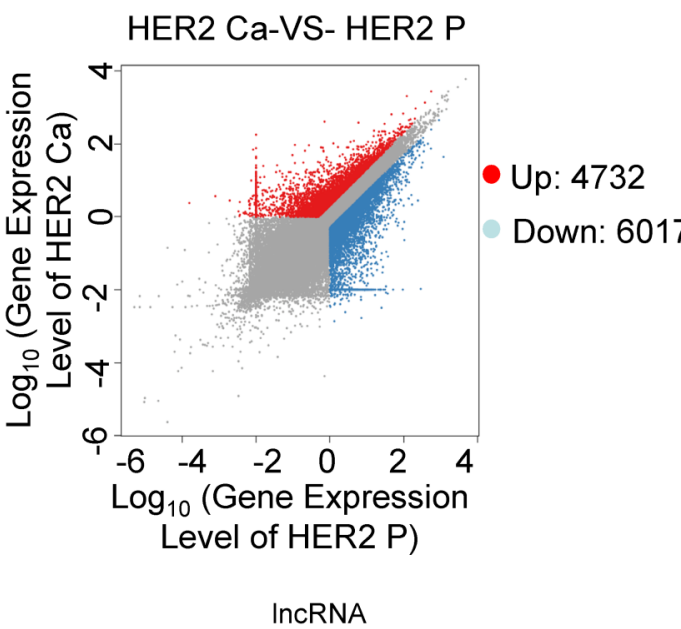

E

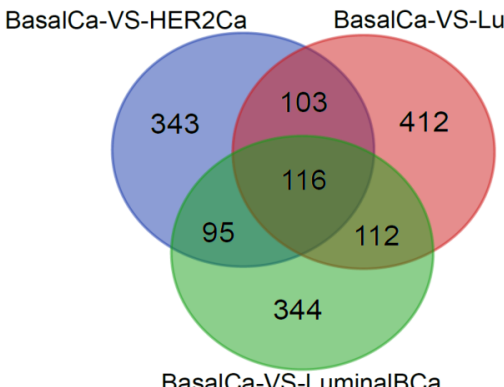

Figure 1: Differential expression analysis of four subtypes of BCs by RNA-sequencing data. A Up-regulated and downregulated genes in Luminal A subtype (Luminal A Ca) compared with adjacent tissue (Luminal A P) (n=3). B Up-regulated and down-regulated genes in Luminal B subtype (Luminal B Ca) compared with adjacent tissue (Luminal B P) $(n=3)$. C Up-regulated and down-regulated genes in HER2 subtype (HER2 Ca) compared with adjacent tissue (HER2 P) $(n=3)$. D Up-regulated and down-regulated genes in Basal subtype (Basal Ca) compared with adjacent tissue (Basal $P$ ) ( $n=3$ ). E Venn diagram analysis of the significant discrepancy of IncRNA in Basal Ca versus Luminal A Ca and Basal Ca versus Luminal B and Basal Ca versus HER2 Ca after filtering the respective IncRNA of adjacent tissue.

and Her2 receptor. The NOl-seq algorithm [9] identified 9544 differentially expressed genes, 5107 downregulated and 4437 up-regulated in Luminal $A$ tissues compared with adjacent tissues (Figure 1A). It identified 12023 differentially expressed genes, 6075 down-regulated and 5948 up-regulated in Luminal B tissues compared with adjacent tissues (Figure 1B). It identified 10749 differentially expressed genes, 6017 down-regulated and 4732 up-regulated in Her2 tissues compared with adjacent tissues (Figure 1C). In addition, it identified 12020 differentially expressed genes, 5437 down-regulated and 6583 up-regulated in Basal-like cancer tissues compared with adjacent tissues (Figure 1D). 
There is mounting evidence showed that IncRNA are differentially expressed in cancers and involve in regulating cellular metabolism, invasion, metastasis, and drug resistance [10]. To elucidate cancer-related IncRNA in Basal subtype, after filtering out the gene expression of adjacent tissues in different $\mathrm{BCa}$ subtypes, we found that 743 expressed genes had significantly differences in Basal subtype compare with Luminal A subtype. The 667 differentially expressed genes were found to change in Basal subtype compare with Luminal B subtype. Besides, it also identified that 657 expressed genes had observably differences in Basal subtype compare with Her2 subtype (Figure 1E). Given that a deficiency of known driver IncRNA for TNBC, these basal-peculiar IncRNA might provide molecular targets for TNBC.

\section{Candidate IncRNA Screening and Differential Expression Analysis}

To extract IncRNA which involved in the regulation of Basal subtype of $\mathrm{BCa}$, we chose differentially expressed IncRNA which are significantly changes [ $\log _{2}$ (Fold change) $>7.0, \mathrm{p}$-value<0.05] in Basal subtype compared with adjacent tissues. These results showed that a total of 41 IncRNA met this standard (Figure 2A). From these, 5 IncRNA (ITGB2-AS1, P2RY8-OT2, ATP2B4-AS1, ZNF518B-OT1, ARHGAP18-AS1) were up-regulated, while 8 IncRNA (TEX14-OT1, LINC01122, TMEM161B-AS1, DSCR8, ECE1-AS1, LINC00856, RTN4-AS1, LINC01122) were down-regulated in Basal subtype compared with Lunimal A subtype (Figure 2A, Table 1); One IncRNA (TMEM254-AS1) were up-regulated, while 8 IncRNA (MSC-AS1, CKMT2-AS1, CACNA2D4-AS1, WDPCPOT3) were down-regulated in Basal subtype compared with Luminal B subtype (Figure 2A, Table 2); 2 IncRNA (SPATA6-AS1, FAM174B-AS1) were up-regulated, while 13 IncRNA (PIK3CD-AS2, ZNF804A-OT1, OCIAD1-AS1, AN010-AS1, BHLHE40-AS1, CSNK1A1L-AS1, MS4A6A-OT1, GPR18-OT3, MCPH1-AS1, TRNT1-OT1, CLIC5-AS2, IL7-OT1, ANKUB1-OT1) were down-regulated in Basal subtype compared with Her2 positive BCa (Figure 2A, Table 3). In addition, we also found that the expression of 4 IncRNA (LINC01169, C1orf162-OT3, MYH13-OT1, ZEB1-AS1) were increased and the expression of 4 IncRNA (COL4A6-OT3, LINC01087, SPRY4-AS1, HLA-DPA1-OT2) were decreased in Basal subtype in contrast to Luminal A\& Luminal B\& Her2 positive BCa (Figure 2A, Tables 4, 5).

Increasing evidence suggested that IncRNA contributed to target regulation and stability by promoting their targets expression or interacting with microRNA [5]. Kyoto Encyclopedia of Genes and Genomes (KEGG) pathway approach identified that the differently signal pathways induced by abovementioned differentially expressed IncRNA. Importantly, the functional enrichment analysis revealed that "Fat digestion and absorption", "Mineral absorption", "Glycerolipid metabolism", "Glycerophospholipid metabolism", "Viral protein interaction with cytokine and cytokine receptor", "Pancreatic secretion", "Aldosterone synthesis and secretion", "Thiamine metabolism", "Nitrogen metabolism" were activated as the top nine pathways (Figure 2B). These differentially expressed targets were found by IncRNA-mRNA co-expression and colocation pairs, such as CEL, PNLIP, FABP2, SLC26A9, MT1HL1, CHAT, ETNPPL, IL20, CXCL13, MC2R, KCNK9, ALPG, and CA3 genes.

\section{Analysis of Survival-Associated IncRNA}

To explore a role of these identified differentially IncRNA in $\mathrm{BCa}$ clinical prognosis, the survival of invasive TNBC patients was analysed using KaplanMeier plotter (www.kmplot.com). Restrict analysis to subtypes included in ER negative, PR negative, HER2 negative, and intrinsic basal subtype of TNBC. The results showed that high expression of IncRNA (ZEB1AS1 and TMEM254-AS1) had poor relapse-free survival rate (RFS, $n=88, \quad Z E B 1-A S 1: H R=2.11$, $\mathrm{P}=0.012 ; \quad$ TMEM254-AS1:HR=2.58, $\quad \mathrm{P}=0.0016)$ compared with low expression (Figure $3 \mathrm{~A}, \mathrm{Bs}$ ), while high expression of IncRNA (LINC01087, LINC01122 and LINC00856) had positive correlation with RFS ( $\mathrm{n}=88, \quad$ LINC01087:HR=0.48, $\mathrm{P}=0.014 ; \quad$ LINC01122: $\mathrm{HR}=0.48, \quad \mathrm{P}=0.014 ; \quad$ LINC00856: $\mathrm{HR}=0.53, \quad \mathrm{P}=0.03$ ) (Figure 3C-E). Others had no significant differences (data not shown). Therefore, the results indicated that these biomarkers might serve as usable prognostic indicators for clinical diagnosis of TNBC.

\section{Real-Time qPCR Identification of Survival- Associated IncRNA}

To further identify the authenticity of screening IncRNA by RNA-sequence, qRT-PCR analysis showed that the mRNA expressions of the IncRNA, including ZEB1-AS1, TMEM254-AS1 were up-regulated in TNBC tissues compared to adjacent tissues (Figure 4). On the contrary, the mRNA expressions of IncRNA, including LINC01087, LINC01122 and LINC00856 were downregulated in TNBC tissues compared to adjacent tissues. Thus, the qRT-PCR results validated the analysis obtained by RNA-sequence data (Figure 4). 
Table 1. All differentially expressed LncRNA between Basal and Luminal A

\begin{tabular}{ccccc}
\hline Gene_ID & Gene name & $\log _{2}$ (fold change) & $P$-value & Up/Down \\
ENSG00000121101 & TEX14-OT1 & -20.8078917646438 & $5.4 \mathrm{E}-08$ & Down \\
ENSG00000233723 & LINC01122-204 & -26.1344519480268 & $2.33 \mathrm{E}-10$ & Down \\
ENSG00000227039 & ITGB2-AS1-202 & 11.3810088517827 & 0.0002 & Up \\
ENSG00000247828 & TMEM161B-AS1-207 & -20.7055247395713 & $6.06 \mathrm{E}-08$ & Down \\
ENSG00000198054 & DSCR8-203 & -20.8170518734616 & $6 \mathrm{E}-08$ & Down \\
ENSG00000117298 & ECE1-AS1 & -7.53559148833483 & 0.04 & Down \\
ENSG00000182162 & P2RY8-OT2 & 9.34749646789377 & 0.002 & Up \\
ENSG00000058668 & ATP2B4-AS1 & 8.35078206373302 & 0.015 & Up \\
ENSG00000230417 & LINC00856-207 & -20.8733933477574 & $4.73 E-08$ & Down \\
ENSG00000115310 & RTN4-AS1 & -21.0565641381349 & $4.27 \mathrm{E}-08$ & Down \\
ENSG00000233723 & LINC01122-201 & -26.1344519480268 & $2.33 E-10$ & Down \\
ENSG00000178163 & ZNF518B-OT1 & 22.1083636813478 & $1.01 E-08$ & Up \\
ENSG00000146376 & ARHGAP18-AS1 & 24.2799938738262 & $1.11 E-09$ & Up \\
\hline
\end{tabular}

Table 2. All differentially expressed LncRNA between Basal and LuminalB

\begin{tabular}{ccccc}
\hline Gene_ID & Gene name & $\log _{2}$ (fold change) & $P$-value & Up/Down \\
ENSG00000235531 & MSC-AS1-207 & -23.9187405636798 & $2.49 \mathrm{E}-09$ & Down \\
ENSG00000230091 & TMEM254-AS1-202 & 24.9420952623797 & $9.83 \mathrm{E}-10$ & Up \\
ENSG00000247572 & CKMT2-AS1-201 & -25.0487599445589 & $9.92 \mathrm{E}-10$ & Down \\
ENSG00000151062 & CACNA2D4-AS1 & -9.46851642179227 & 0.0234 & Down \\
ENSG00000143951 & WDPCP-OT3 & -26.0461305972269 & $4.08 \mathrm{E}-10$ & Down \\
\hline
\end{tabular}

Table 3. All differentially expressed LncRNA between Basal and HER2

\begin{tabular}{ccccc}
\hline Gene_ID & Gene name & $\log _{2}$ (fold change) & $P$-value & Up/Down \\
ENSGO0000231789 & PIK3CD-AS2-201 & -21.3367059527513 & $7.16 \mathrm{E}-08$ & Down \\
ENSG00000170396 & ZNF804A-OT1 & -7.67094165277261 & 0.031 & Down \\
ENSG00000248256 & OCIAD1-AS1-201 & -8.85184022471439 & 0.041 & Down \\
ENSG00000160746 & ANO10-AS1 & -25.4945428788784 & $1.06 \mathrm{E}-09$ & Down \\
ENSG00000235831 & BHLHE40-AS1-202 & -10.0167381091245 & 0.002 & Down \\
ENSG00000180138 & CSNK1A1L-AS1 & -21.4976470307641 & $3.64 \mathrm{E}-08$ & Down \\
ENSG00000110077 & MS4A6A-OT1 & -10.8075270059552 & 0.007 & Down \\
ENSG00000125245 & GPR18-OT3 & -24.1973311647625 & $3.5 \mathrm{E}-09$ & Down \\
ENSG00000249889 & MCPH1-AS1-203 & -20.8687635700174 & $7.94 \mathrm{E}-08$ & Down \\
ENSG00000072756 & TRNT1-OT1 & -7.15151468484095 & 0.014 & Down \\
ENSG00000112782 & CLIC5-AS2 & -11.3502609746371 & 0.004 & Down \\
ENSG00000104432 & IL7-OT1 & -11.5748489769009 & 0.004 & Down \\
ENSG00000206199 & ANKUB1-OT1 & -9.54602529298167 & 0.022 & Down \\
ENSG00000132122 & SPATA6-AS1 & 22.2757907234644 & $1.54 \mathrm{E}-08$ & Up \\
ENSG00000185442 & FAM174B-AS1 & 21.8581606085617 & $2.44 E-08$ & Up \\
\hline
\end{tabular}

Table 4. All differentially expressed LncRNA between Basal and Luminal A/LuminalB

\begin{tabular}{ccccc}
\hline Gene_ID & Gene name & $\log _{2}$ (fold change) & $P$-value & Up/Down \\
ENSG00000197565 & COL4A6-OT3 & -8.80848274866248 & 0.035 & Down \\
ENSG00000224559 & LINC01087-201 & -9.44945635288289 & 0.002 & Down \\
ENSG00000259471 & LINC01169-201 & 22.6810754259996 & $5.18 E-09$ & Up \\
ENSG00000143110 & C10rf162-OT3 & 22.2173016660523 & $8.88 E-09$ & Up \\
ENSG00000006788 & MYH13-OT1 & 23.9679626196533 & $1.49 E-09$ & Up \\
\hline
\end{tabular}

Table 5. All differentially expressed LncRNA between Basal and HER2/Luminal A/LuminalB

\begin{tabular}{ccccc}
\hline Gene_ID & Gene name & $\log _{2}$ (fold change) & $P$-value & Up/Down \\
ENSG00000148516 & ZEB1-AS1 & 22.8255726317403 & $9.45 E-09$ & Up \\
ENSG00000231185 & SPRY4-AS1-202 & -24.4484774565741 & $2.57 \mathrm{E}-09$ & Down \\
ENSG00000231389 & HLA-DPA1-OT2 & -28.2388156823631 & $6.88 \mathrm{E}-11$ & Down \\
\hline
\end{tabular}

B

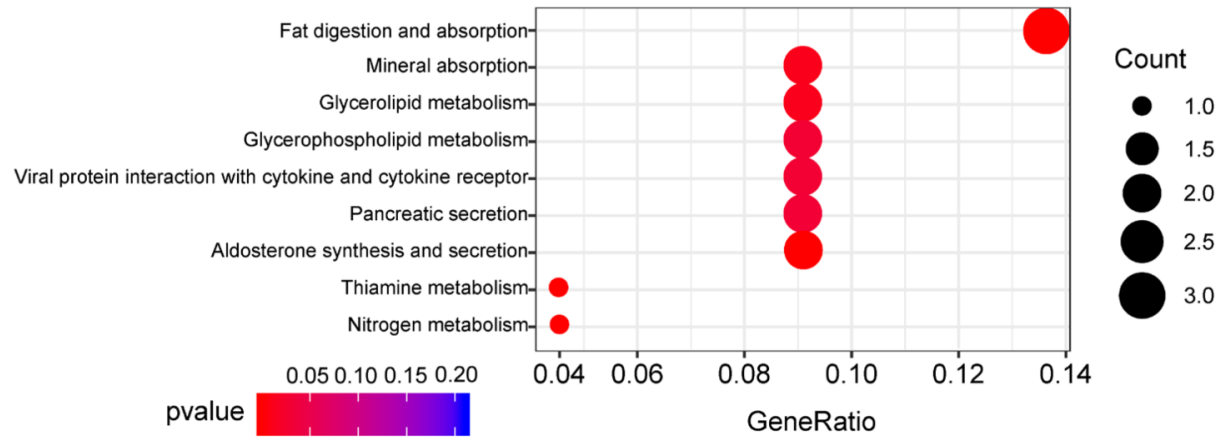

Figure 2: Screening of cancer-associated IncRNA in Basal subtype. A Differential IncRNA analysis was listed for overexpressed and deficient IncRNA in Basal versus Luminal A (Table 1), Basal versus Luminal B (Table 2), Basal versus HER2 (Table 3), Basal versus Luminal A versus Luminal B (Table 4), Basal versus Luminal A versus Luminal B versus HER2 (Table 5). B Enrichment differential pathways were shown by KEGG analysis. 
A

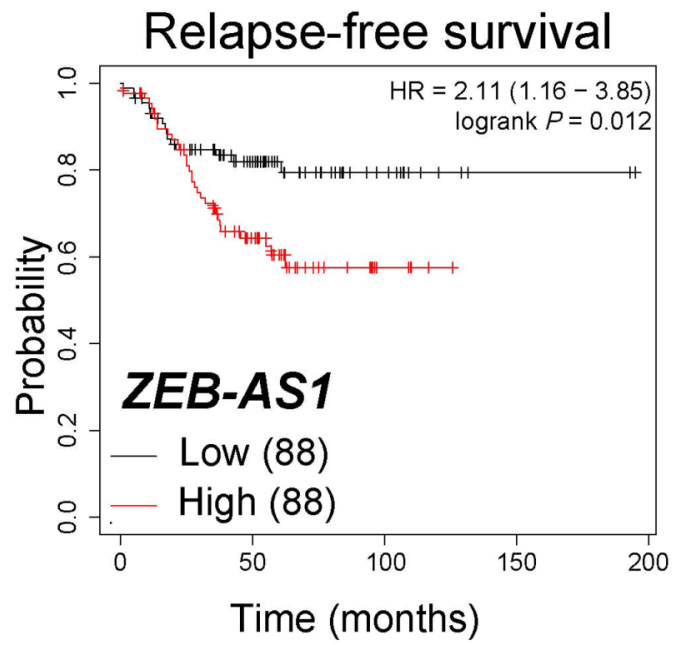

C

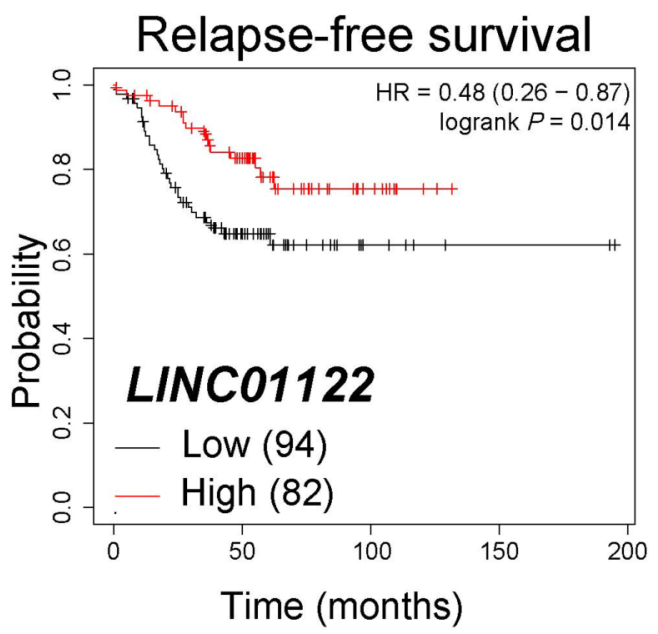

$E$

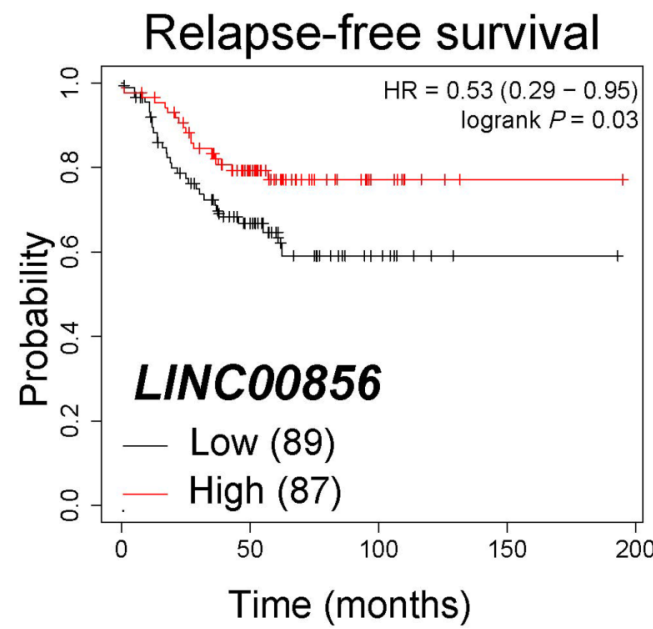

B

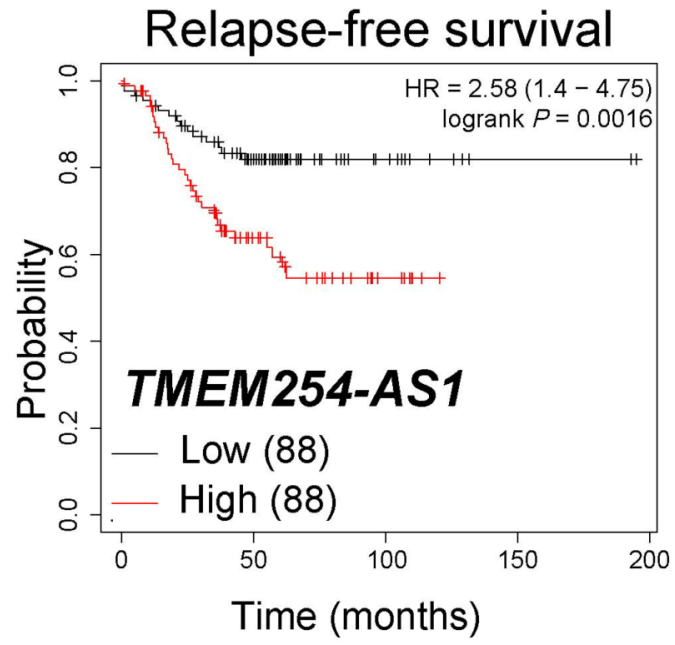

$\mathrm{D}$

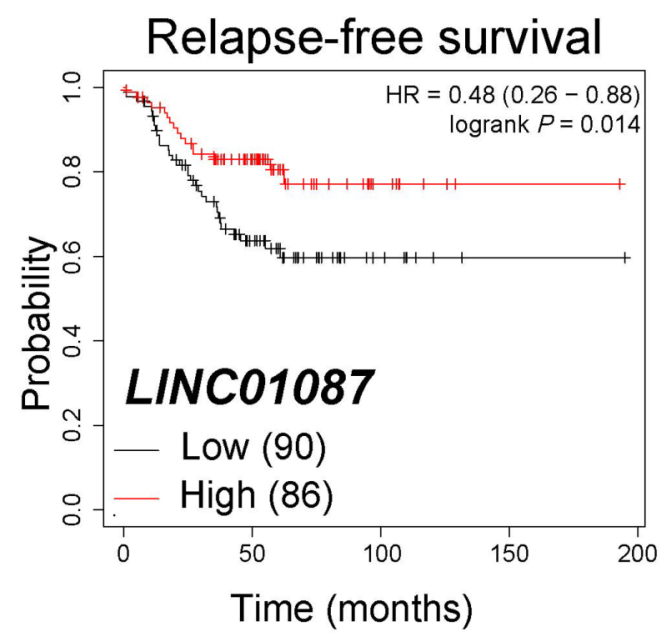

Figure 3: The correlation between the RNA expression levels of identified IncRNA and relapse-free survival in Basal subtype BCa from Kaplan-Meier plotter dataset. Kaplan-Meier analysis of relapse-free survival of BCa patients from 'high' and 'low' mRNA expression levels of (A) ZEB-AS1, (B) TMEM254-AS1, (C) LINC01087, (D) LINC01122 and (E) LINC00856. 
A

B
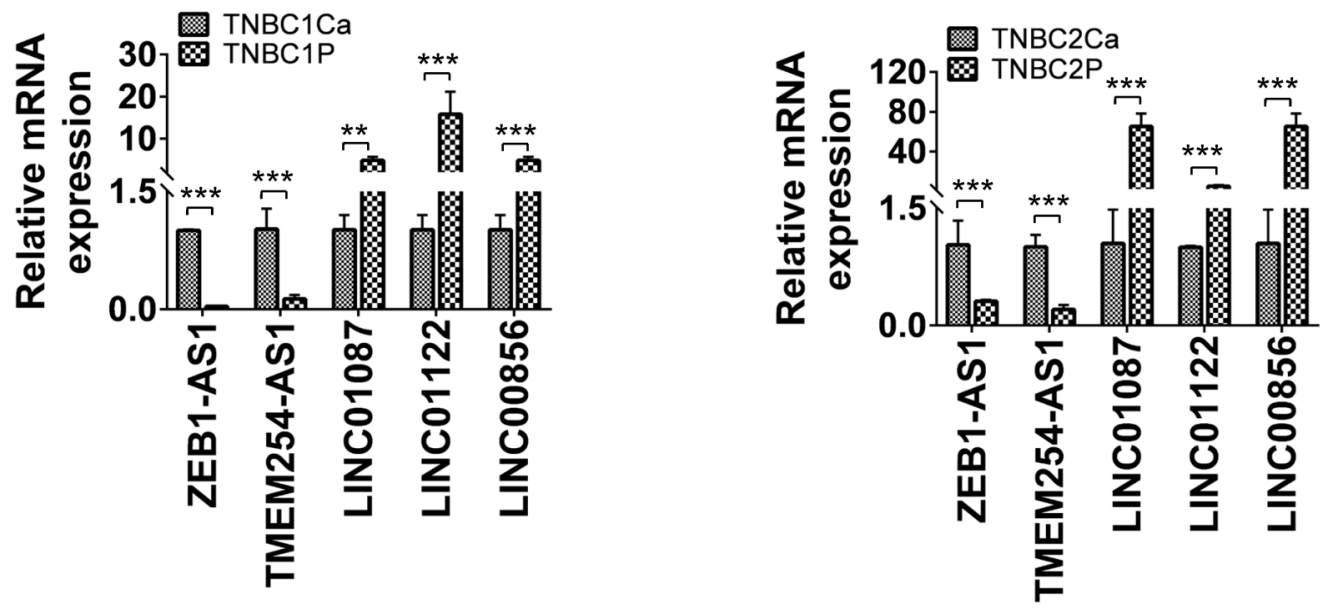

C

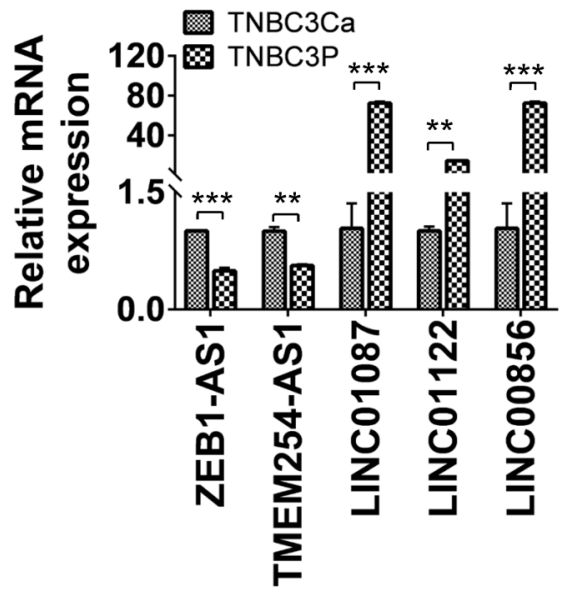

Figure 4: QRT-PCR analysis was used to identify the expression of InRNA. Each experiment was performed in triplicate and data was represented as mean \pm S.D. One-Way ANOVA and Dunnett's multiple comparison test were used to analyse the data $\left({ }^{\star} P<0.05,{ }^{* *} P<0.01,{ }^{* * *} P<0.001\right)$.

\section{DISCUSSION}

TNBC is a heterogeneous tumor, that has high invasiveness, high mitotic rates and chemotherapy resistance [11]. Our data provided 5 potential biomarkers for TNBC. Previous studies showed that ZEB1-AS1 could promote tumor progression of hepatocellular carcinoma by up-regulating its target ZEB1 [12]. Besides, the high expression of ZEB1-AS1 had correlated with poor outcome of malignancy including glioma and prostate cancer [13, 14]. It was found that TMEM254-AS1 contributed to be upregulated in persistence cells of neoadjuvant chemotherapy for TNBC by single-cell IncRNA transcriptome, that might serve as novel therapeutic target against TNBC [15]. Previous studies suggested that LINC01087 had high expression levels in BCa. LINC01087 was found to be significantly upregulated in Luminal subtype of $\mathrm{BCa}$ in contrast to TNBC subtype [16]. However, its molecular mechanism remains unknown on $\mathrm{BCa}$. In addition, the correlation and regulatory function between LINC01122 or LINC00856 and cancers has not been reported. Due to the limitation of tissue sample size for RNA-sequencing, further identification needs to be analysed through expanding sample size.

In conclusion, we identified that 5 novel IncRNAs, including ZEB1-AS1, TMEM254-AS1, LINC01087, LINC01122 and LINC00856 contributed to the progression of invasive TNBC. The overexpression of ZEB1-AS1 or TMEM254-AS1 was related to poor 
prognosis of TNBC patients, while downregulation of LINC01087, LINC01122 and LINC00856 was associated with poor outcome of TNBC patients. In brief, this study identifies novel IncRNAs in TNBC progression and could be potential therapeutic targets against TNBC.

\section{Data Availability Statements}

All data that support the findings of this study are available from the corresponding author upon reasonable request.

\section{ACKNOWLEDGEMENTS}

We thank the Cancer Genome Atlas and KaplanMeier Plotter for providing their data platform. This work was supported by grants from the China Postdoctoral Science Foundation Grant 2019M660232 (Lin Gao), the National Natural Science Foundation of China 82002929 (Lin Gao), Basic and Applied Basic Research Foundation of Guangdong Province [2020A1515111072 (Lin Gao), 2019B151512003 (Chang Zou), 2020B1515120032 (Chang Zou)], the Science and Technology Foundation of Shenzhen JCYJ20190806154610953 (Lin Gao), JCYJ20180305164128430 (Chang Zou), the International Cooperation Foundation of Shenzhen GJHZ20180928171602104 (Chang Zou), the Shenzhen Economic and Information Committee "Innovation Chain and Industry Chain" integration special support plan project 20180225112449943 (Chang Zou), the Shenzhen Public Service Platform on Tumor Precision Medicine and Molecular Diagnosis (Chang Zou), the Ministry of Science and Technology of the People's Republic of China (No. 2018ZX09201018), the National Natural Science Foundation of China(No. 81803183), the Shenzhen Key Medical Discipline Construction Fund (SZXK053).

\section{CONFLICT OF INTERESTS}

The authors declared no competing interests.

\section{AUTHOR CONTRIBUTIONS}

Lin Gao designed the project and wrote the manuscript. Jinquan Xia, Malin Hong and Jingyi Huang were responsible to analysed for RNA-seq data. Pan Zhao performed the experiments. Yong Dai, Wenbin Zhou and Qinhe Yang provided BCa samples. Li Fu and Jigang Wang supported clinical and pathological information. Chang Zou designed and supervised this project and revised the manuscript.

\section{SUPPLEMENTAL TABLE}

The supplemental Table can be downloaded from the journal website along with the article.

\section{REFERENCE}

[1] Jiang YZ, Ma D, Suo C, Shi J, Xue M, Hu X, et al. Genomic and Transcriptomic Landscape of Triple-Negative Breast Cancers: Subtypes and Treatment Strategies. Cancer Cell 2019; 35(3): 428-440 e425.

[2] Jiang $Y Z$, Liu $Y R, X u X E$, Jin $X, H u X, Y u K D$, et al. Transcriptome Analysis of Triple-Negative Breast Cancer Reveals an Integrated mRNA-IncRNA Signature with Predictive and Prognostic Value. Cancer Res 2016; 76(8): 2105-2114.

https://doi.org/10.1158/0008-5472.CAN-15-3284

[3] de la Mare JA, Contu L, Hunter MC, Moyo B, Sterrenberg JN Dhanani KC, Mutsvunguma LZ, Edkins AL. Breast cancer: current developments in molecular approaches to diagnosis and treatment. Recent Pat Anticancer Drug Discov 2014; 9(2): 153-75.

https://doi.org/10.2174/15748928113086660046

[4] Huang JZ, Chen M, Chen, Gao XC, Zhu S, Huang H, et al. A Peptide Encoded by a Putative IncRNA HOXB-AS3 Suppresses Colon Cancer Growth. Mol Cell 2017; 68(1): 171-184 e176. https://doi.org/10.1016/j.molcel.2017.09.015

[5] Anastasiadou E, Jacob LS, Slack FJ. Non-coding RNA networks in cancer. Nat Rev Cancer 2018; 18(1): 5-18. https://doi.org/10.1038/nrc.2017.99

[6] Niknafs YS, Han S, Ma T, Speers C, Zhang C, WilderRomans $\mathrm{K}$, et al. The IncRNA landscape of breast cancer reveals a role for DSCAM-AS1 in breast cancer progression. Nat Commun 2016; 712791. https://doi.org/10.1038/ncomms12791

[7] Gupta RA, Shah N, Wang KC, Kim J, Horlings HM, Wong DJ et al. Long non-coding RNA HOTAIR reprograms chromatin state to promote cancer metastasis. Nature 2010; 464 (7291): 1071-1076 https://doi.org/10.1038/nature08975

[8] Robinson MD, McCarthy DJ, Smyth GK. edgeR: a Bioconductor package for differential expression analysis of digital gene expression data. Bioinformatics 2010; 26(1): 139-140. https://doi.org/10.1093/bioinformatics/btp616

[9] Soneson C, Delorenzi M. A comparison of methods for differential expression analysis of RNA-seq data. BMC Bioinformatics 2013; 14: 91.

https://doi.org/10.1186/1471-2105-14-91

[10] Liu K, Gao L, Ma X, Huang JJ, Chen J, Zeng L, et al. Long non-coding RNAs regulate drug resistance in cancer. Mol Cancer 2020; 19(1): 54 https://doi.org/10.1186/s12943-020-01162-0

[11] Moens S, Zhao P, Baietti MF, Marinelli O, Van Haver D, Impens $\mathrm{F}$, et al. The mitotic checkpoint is a targetable vulnerability of carboplatin-resistant triple negative breast cancers. Sci Rep 2021; 11(1): 3176 https://doi.org/10.1038/s41598-021-82780-6

[12] Li T, Xie J, Shen C, Cheng D, Shi Y, Wu Z, et al. Upregulation of long noncoding RNA ZEB1-AS1 promotes tumor metastasis and predicts poor prognosis in hepatocellular carcinoma. Oncogene 2016; 35(12): 1575-1584. https://doi.org/10.1038/onc.2015.223

[13] Su W, Xu M, Chen X, Chen N, Gong J, Nie L, et al. Long noncoding RNA ZEB1-AS1 epigenetically regulates the expressions of ZEB1 and downstream molecules in prostate cancer. Mol Cancer 2017; 16(1): 142. https://doi.org/10.1186/s12943-017-0711-y 
[14] Lv QL, Hu L, Chen SH, Sun B, Fu ML, Qin CZ, et al. A Long Noncoding RNA ZEB1-AS1 Promotes Tumorigenesis and Predicts Poor Prognosis in Glioma. Int J Mol Sci 2016; 17(9). https://doi.org/10.3390/ijms17091431

[15] Shaath H, Vishnubalaji R, Elango R, Khattak S, Alajez N.M Single-cell long noncoding RNA (IncRNA) transcriptome implicates MALAT1 in triple-negative breast cancer (TNBC) resistance to neoadjuvant chemotherapy. Cell Death Discov $2021 ; 7(1): 23$.

https://doi.org/10.1038/s41420-020-00383-y

[16] De Palma FDE, Del Monaco V, Pol JG, Kremer M, D'Argenio $\mathrm{V}$, Stoll G, et al. The abundance of the long intergenic noncoding RNA 01087 differentiates between luminal and triplenegative breast cancers and predicts patient outcome. Pharmacol Res 2020; 161: 105249.

Received on 05-04-2021

Accepted on 20-05-2021

Published on 25-05-2021

https://doi.org/10.30683/1929-2279.2021.10.01

(C) 2021 Gao et al.; Licensee Neoplasia Research.

This is an open access article licensed under the terms of the Creative Commons Attribution Non-Commercial License (http://creativecommons.org/licenses/by-nc/3.0/) which permits unrestricted, non-commercial use, distribution and reproduction in any medium, provided the work is properly cited. 